\title{
Aptamer selection by high-throughput sequencing and informatic analysis
}

\author{
Shawn Hoon ${ }^{1}$, Bin Zhou ${ }^{3,4}$, Kim D. Janda ${ }^{3,4}$, Sydney Brenner ${ }^{1,2}$, and Jonathan Scolnick ${ }^{1,2}$ \\ ${ }^{1}$ Molecular Engineering Lab, Agency for Science Technology and Research, Singapore and ${ }^{2}$ Department of Chemical \\ Physiology, The Scripps Research Institute, La Jolla, CA, ${ }^{3}$ Departments of Chemistry and Immunology and \\ Microbial Science, The Skaggs Institute for Chemical Biology, and ${ }^{4}$ The Worm Institute for Research and Medicine
}

BioTechniques 51:413-416 (December 2011) doi 10.2144/000113786

Keywords: aptamer; high throughput sequencing; informatics

Supplementary material for this article is available at www.BioTechniques.com/article/113786

Traditional methods for selecting aptamers require multiple rounds of selection and optimization in order to identify aptamers that bind with high affinity to their targets. Here we describe an assay that requires only one round of positive selection followed by high-throughput DNA sequencing and informatic analysis in order to select high-affinity aptamers. The assay is flexible, requires less hands on time, and can be used by laboratories with minimal expertise in aptamer biology to quickly select high-affinity aptamers to a target of interest. This assay has been utilized to successfully identify aptamers that bind to thrombin with dissociation constants in the nanomolar range.

Aptamers are nucleic acid oligomers that are synthesized to bind to target molecules with high affinity and selectivity $(1,2)$. Aptamers have been described that bind to small molecules, proteins, and cells making them important reagents with a wide range of uses including medical diagnostics, imaging and therapeutics (2-6).

Aptamers against a particular target are most often identified through a process referred to as systematic evolution of ligands by exponential enrichment $(\operatorname{SELEX})(1,2)$. In the SELEX process, a library of randomized nucleic acid sequences, typically $40-60$ bases in length, is exposed to a target molecule. Those sequences that bind the target are retained, amplified, and utilized in further rounds of selection. Following multiple rounds of selection, individual aptamers are cloned and sequenced in order to identify the highest affinity binding aptamers from the pool. These lead aptamers are further modified through a series of deletions in order to optimize the aptamer sequence for the highest affinity to the target. While powerful, the overall SELEX process is time consuming, taking weeks to move from random library to optimized aptamer.

Recently Cho et. al. (7) and Kupakuwana et. al. (8) published studies that utilized highthroughput sequencing to shorten the initial aptamer selection time. However, while both groups were able to identify aptamers that bind to the intended target, they either relied on multiple rounds of selection and sequencing (7) or limited the flexibility available in the sequence space by only analyzing fixed-length sequences (8).

Here we describe a method for selecting high-affinity DNA aptamers based on a single round of selection followed by highthroughput sequencing and bioinformatic analysis. Aptamer sequences were identified by selecting variable $\mathrm{k}$-mer length sequences that are enriched in the sequenced library. Using this method to identify DNA aptamers that bind to thrombin, we found both known and novel thrombin binding aptamers. Our method, Aptamer Selection by K-mer Analysis of Sequences (ASKAS) provides a tool for researchers to discover high-affinity aptamers of varying lengths with minimal hands on time.

\section{Materials and methods \\ Aptamer library}

A single stranded DNA oligonucleotide library was made by Integrated DNA Technologies (Coralville, Iowa, USA). The library consisted of 33 random bases flanked by fixed regions corresponding to the Illumina GA adapter sequences 5' - ACACTCTTTCCCTACACGACGCTCTTCCGATCT-3' and 5' AGATCGGAAGAGCTC-3'. These flanking sequences form a stable $13 \mathrm{bp}$ stem so that the random library is presented as a 33 bp hairpin. The library had a theoretical diversity of $7 \times 10^{19}$ sequences. Prior to use, the library was diluted in thrombin binding buffer (9) consisting of $50 \mathrm{mM}$ Tris $\mathrm{pH} 7.5$, $100 \mathrm{mM} \mathrm{NaCl}, 1 \mathrm{mM} \mathrm{MgCl}$. A positive control aptamer described in Tasset et al. (9), 5'-GAGTCCGTGGTAGGGCAGGTTGGGGTGACTTCGTGGAA -3' was made and flanked by the same fixed regions as the random library.

Thrombin preparation

1790 U of Human Thrombin (Sigma-Aldrich, St. Louis, MO, USA) was diluted in $130 \mu \mathrm{l}$ of PBS giving a concentration of $11.9 \mu \mathrm{M}$. $112 \mu \mathrm{l}$ was then added to $160 \mu \mathrm{l}$ of pre-washed epoxy coated magnetic beads (Life Technologies, Carlsbad, CA, USA) with 48 ul of $3 \mathrm{M}$ $\left(\mathrm{NH}_{4}\right)_{2} \mathrm{SO}_{4}$ and was incubated overnight at $37^{\circ} \mathrm{C}$ followed by washing three times in thrombin binding buffer. The beads were resuspended in $160 \mu \mathrm{l}$ of thrombin binding buffer and $10 \mu \mathrm{l}$, approximately 80 pmoles of thrombin, were used for the aptamer selection. Negative selection beads were treated as above, but without the presence of thrombin protein.

\section{Aptamer selection}

Approximately $10^{12}$ molecules of aptamer library and 6000 copies of positive control aptamer were separately heated to $95^{\circ} \mathrm{C}$ 
followed by $5 \mathrm{~min}$ on ice and a $5 \mathrm{~min}$ incubation at room temperature. The two were then combined for selection in $50 \mu \mathrm{l}$ of thrombin binding buffer plus $0.1 \%$ BSA. A negative selection was performed by adding the library to the blank beads for $30 \mathrm{~min}$. Following the negative selection, a magnet was used to capture the beads and the solution containing unbound aptamer was transferred to a tube containing thrombincoated beads. Aptamers were incubated at room temperature for $30 \mathrm{~min}$ with the thrombin-coated beads at which point the unbound aptamers were removed and fresh buffer was added to the tube. Two more 30 min incubations followed by buffer exchanges were performed. After the third incubation, beads were quickly washed in buffer one more time before performing a PCR directly on the beads. Twelve cycles of PCR amplification was conducted using KOD Hot Start polymerase (Merck Biosciences, Gibbstown, NJ, USA) with Illumina amplification primers 5' - AATGATACGGCGACCACCGAGATCTACACTCTTTCCCTACACGACGCTCTTCCGATCT $-3^{\prime}$ and $5^{\prime}$ - CAAGCAGAAGACGGCATACGAGCTCTTCCGATCT -3 ' that hybridize to the fixed sequence of the aptamers. The PCR product was gel extracted and quantitated on a Bioanalyzer (Agilent Technologies, Santa Clara, CA, USA) using the High Sensitivity DNA kit. The library was then sequenced on an Illumina GA2x (Illumina, San Diego, CA, USA) following the manufacturer's protocols for single pass 36 cycle sequencing using the Illumina Genomic DNA Sequencing Primer, 5' ACACTCTTTCCCTACACGACGCTCTTCCGATCT 3'. A second aptamer library that had not been selected for thrombin binders was also sequenced following the same methodology.

Sequence analysis

Sequence reads were preprocessed first by removing sequences that were $>80 \%$ homopolymeric. Sequences that did not contain any aptamer sequence were also removed. After analyzing the results of the positive control aptamer, further analysis was performed with a data set in which all sequences containing 13 contiguous bases matching the positive control aptamer sequence were removed. K-mer analysis was conducted using the freely available Tallymer software (10). K-mer values of $15-33$ were used.

K-mers were clustered ( $70 \%$ identity) using the cluster_seq (http://code.google. $\mathrm{com} / \mathrm{p} /$ biopieces) program which is driven by the uclust clustering engine (11). The 70\% identity level was chosen for clustering in order to compress the top ten sequences down to five for the determination of dissociation constants by surface plasmon resonance.

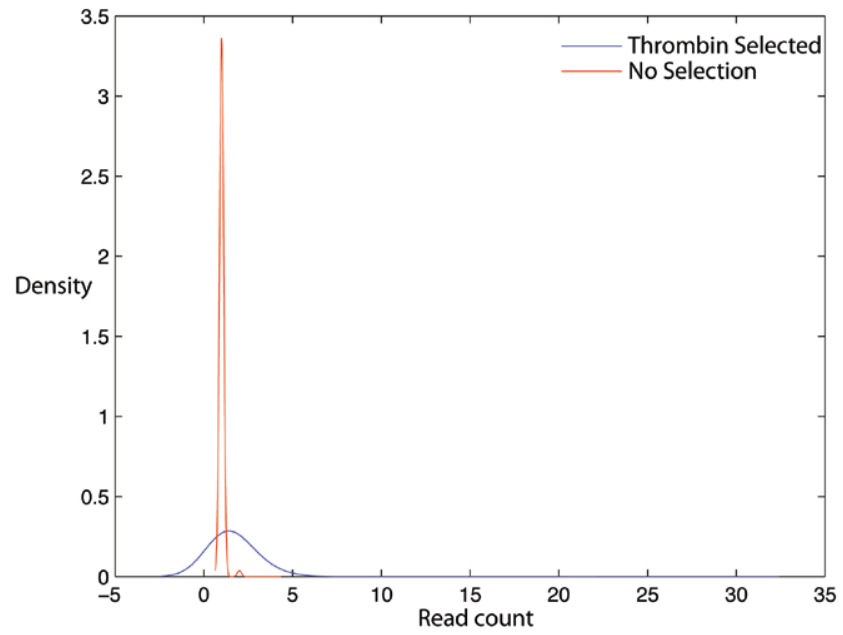

Figure 1. Distribution of reads counts from the aptamer libraries. In the naïve library (red) $98.9 \%$ of the 33mer sequences are unique. The distribution of the Thrombin selected library (blue), by contrast, is shifted to the right suggesting that exposure to Thrombin did select for certain sequences. The continuous density estimate was generated using the Matlab function ksdensity (Kernel smoothing density estimate) with normal kernel.

Dissociation constants of aptamers and thrombin were measured using surface plasmon resonance (Biacore Flexchip, GE Life Sciences, Piscataway, NJ, USA). A streptavidin coated chip was spotted with biotinylated aptamers (IDT) and 50mM Thrombin in thrombin binding buffer was used to make the measurements.

\section{Quantitative PCR}

Quantitative PCR was carried out using the Quantitect Sybr Green PCR kit (Qiagen) and analyzed on an ABI 7900 FAST (Life Technologies). Primers used were the Illumina amplification primers described above.

\section{Results and discussion}

Aptamer selection procedure

Traditional SELEX experiments require multiple rounds of binding aptamers to the target in order to increase the probability that the small number of individually cloned sequences will represent high-affinity aptamers and not artifacts of the SELEX experimental design. In contrast, we chose to utilize high-throughput sequencing which provides millions of sequences as a readout of the selection process.

An aptamer library was prepared and subjected to selection as described in Material and Methods. Previous data from Berezovski et al. (12) showed that three rounds of partitioning was sufficient to increase the overall library affinity to the target by 5 orders of magnitude. Our own preliminary experiments also suggested that three washes was sufficient to remove much of the non thrombin binding aptamer (data not shown), hence, three washes of the aptamer library bound to thrombin were performed. The selected aptamers were amplified and sequenced as described in Materials and Methods.

Two libraries were sequenced; the library selected for thrombin binding aptamers and a second non-selected (naïve) library as a test for the randomization of the original aptamer pool. We obtained sequencing reads from both libraries and after preprocessing the sequences to remove poor quality sequencing reads (see Materials and Methods), 11.5M and $15 \mathrm{M}$ reads respectively were used for further analysis.

\section{Selection for thrombin-binding} aptamers

An initial analysis was performed to determine whether or not our aptamer selection procedure altered the distribution of sequences obtained from the two libraries. $98.9 \%$ of the sequences in the naive library were found to be unique giving us confidence that our initial aptamer pool did not have any strong sequence biases. After filtering out the positive control sequences (see Materials and Methods), we compared the distribution of sequences from the naive library to that of the thrombin selected library. Figure 1 shows a clear shift in the distributions such that more aptamers were sequenced multiple times in the selected library compared with the naïve, unselected library. For example, in the naive library only one $33 \mathrm{mer}$ was sequenced five times, whereas in the thrombin selected library 84,62733 mer sequences were counted five or more times with one sequence appearing 31 times. The larger number of repeated sequences in the selected library exists despite the higher number of overall sequencing reads in the naïve library. These data suggest that our selection protocol did enrich for sequences that bind to thrombin. 
Enrichment of the positive control aptamer was next investigated. Approximately 6000 copies of the positive control aptamer, representing $6 \times 10^{-7} \%$ of the total aptamer sequences in the library (6000 copies out of $10^{12}$ molecules), were initially added to the random library. Without selection, the positive control aptamer was sequenced 90 times ( 6 $\times 10^{-4} \%$ of total sequencing reads). Following selection against thrombin, the positive control aptamer was sequenced 5925 times representing $\sim 0.05 \%$ of the sequences. When normalized for total read count, this represents an 88.5 fold enrichment of the known thrombin binding sequence. These data provide further evidence that our selection procedure did select for aptamers that bind to the thrombin protein.

While the positive control sequences did show that our selection procedures worked it is also important to note that the sequence is represented approximately 1000 times more frequently in the sequencing reads than expected in the naive library. There are a number of factors that potentially contributed to this overrepresentation. We directly investigated the possibility of PCR bias by comparing the amplification of the positive control aptamer to the bulk aptamer library by qPCR analysis. Indeed, we did find that the positive control sequence amplified better than the aptamer library (Ct values of $30.57+/-0.32$ compared with $35.58+/-0.4$, $\left.P<4 \times 10^{-10}\right)$. Based on this result, we believe there may also be a positive bias in amplification on the Illumina flow cell such that this particular sequence becomes overrepresented on the sequencing instrument. It is not clear if this bias is due to the sequence composition or the shorter sequence length of the positive control compared with the aptamer library; however, the nucleotide distribution over all of the sequencing reads in both the naive and selected libraries is $25 \%$ for each nucleotide suggesting that the length of positive control aptamer may be the most important factor.

Identifying aptamers via $\mathrm{K}$-mer analysis Following the SELEX process, aptamers are frequently modified by making a series of deletions in order to develop aptamers that bind most tightly to the target. Rather than making a series of physical deletions to the 33 mer sequences we obtained, $\mathrm{k}$-mer analysis was performed in order to identify subsequences within the 33 mers that were responsible for binding to thrombin. The frequency distribution of shorter k-mer length sequences was therefore analyzed to find overrepresented sequences in the data set, as those sequences may represent the actual thrombin binding sequences. For these analyses we first filtered out the positive control sequences (see Materials and Methods).

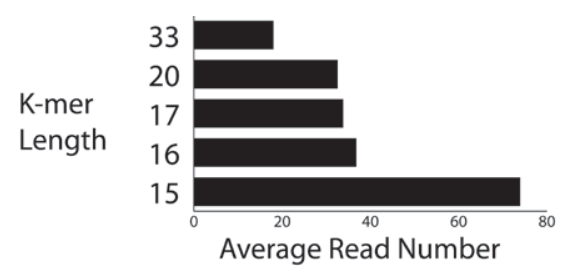

Figure 2. Average sequence counts of the top ten most represented sequences for each k-mer length. The average sequence count increases as the k-mer length decreases. After small increases between $k=33$ and $k=16$, there is a 2 fold increase in the average at $k=15$.

A k-mer analysis was performed using the Tallymer program to elucidate the representation of k-mers, where k ranged from 15 to 33 bases. As expected, the top 10 most frequent sequences from each $\mathrm{k}$-mer length increased in read frequency as the k-mer size decreased (Figure 2). For example, at $\mathrm{k}=20$ the top 10 sequences averaged 32.4 reads while at $\mathrm{k}$ $=16$, the average was 36.8 reads. At $\mathrm{k}=15$, there was a jump in sequence representation; the top 1015 mer sequences were counted an average of 73.9 times. Given the doubling of sequence representation between $15 \mathrm{mer}$ and 16 mer sequences, we focused on these two sets of sequences for further analysis.

Sequence selection and dissociation constant measurements

A cluster analysis of the 15 mer and 16 mer sequences was performed in order to group the most similar sequences (see Materials and Methods). The five most represented sequences found in separate clusters were selected for further testing.

Surface Plasmon Resonance (SPR) was utilized to measure the dissociation constant between each of the selected aptamers and thrombin. The results are shown in Table 1. Of the 10 aptamers selected for testing, only one did not show any measurable binding by the SPR assay. All other aptamers tested had $\mathrm{K}_{\mathrm{d}}$ values in the nano to micro molar range. Two 15 mers, including an aptamer sequence previously identified by Bock et. al.(5), had $\mathrm{K}_{\mathrm{d}}$ 's measured in the tens of $\mathrm{nM}$ range (raw traces in Figure S1). These $\mathrm{K}_{\mathrm{d}}$ measurements are similar to those found by multiple rounds of aptamer selection suggesting that a single round of aptamer selection followed by high throughput sequencing and bioinformatic analysis can be used to substitute for the more time consuming SELEX method of aptamer selection.

\section{Constraints of ASKAS}

While thrombin binding aptamers, including a novel one, were identified by the ASKAS method, future experimentation will be required to further optimize the ASKAS pipeline. For example, read counts did

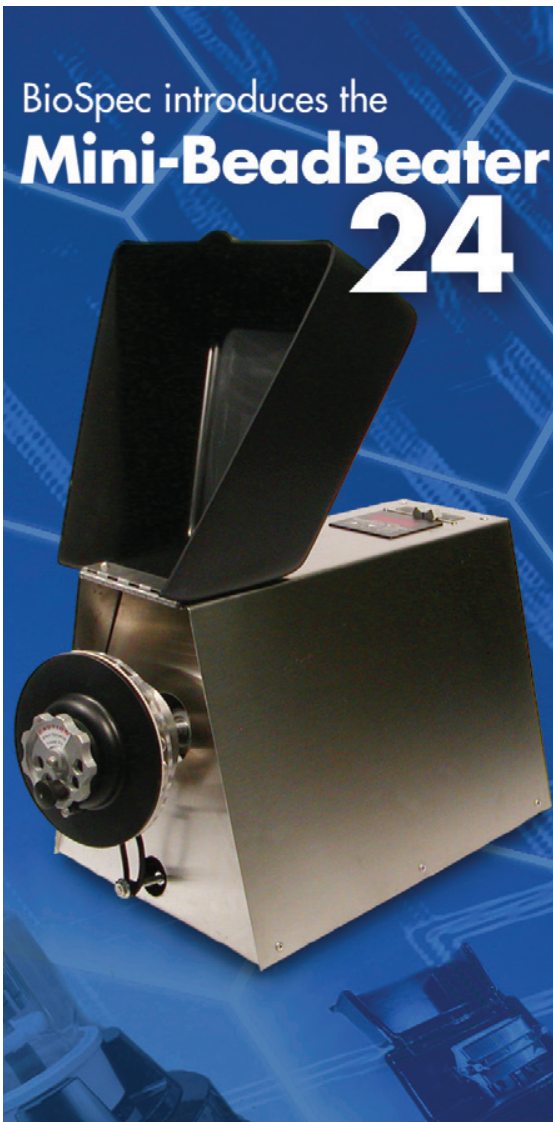

The Mini-Beadbeater-24 disrupts microorganisms and tissue

preparations with better than 95 percent efficiency. Nucleic acids and proteins are isolated with the highest possible yields. Cells are disrupted quickly and safely in the sealed $2 \mathrm{ml}$ microvials containing small beads. No cleaning between samples is required. The apparatus has a small footprint and is essentially maintenance free.

- Uses proven, more efficient near horizontal vial shaking motion.

- $24-2.0 \mathrm{ml}$ microvials capacity with removable vial-holding cassette.

- Variable shaking speed:2000 - 3450

strokes/min

- Digital 5 minute timer with auto reset.

- No imposed motor cool-downtime between each sample run.

- Best price.

BSE BIOSPEC PRODUCTS

P.O. Box 788 Bartlesville, OK 74005

800-617-3363 www.biospec.com 
Table 1. Dissociation Constant Measurements for Selected Aptamers

\begin{tabular}{|c|c|c|c|c|c|}
\hline $\begin{array}{c}15 \text { mer } \\
\text { Sequence }\end{array}$ & Counts & $\mathrm{K}_{\mathrm{d}}(\mathrm{M})$ & $\begin{array}{c}16 \text { mer } \\
\text { Sequence }\end{array}$ & Counts & $K_{d}(M)$ \\
\hline ggttggtttggttgg & 98 & $1.99 \times 10^{-7}$ & tggtttggttggtaga & 40 & $3.23 \times 10^{-7}$ \\
\hline ttggtttggttggtt & 80 & Binding Not Detected & ggttggtttggttggt & 38 & $1.0 \times 10^{-6}$ \\
\hline ggttggtgtggttgg* & 79 & $2.6 \times 10^{-8}$ & ggtttggtttggttgg & 37 & $1.6 \times 10^{-7}$ \\
\hline ggttgggttggttgg & 64 & $4.49 \times 10^{-6}$ & ggtatggtttggttgg & 37 & $1.25 \times 10^{-7}$ \\
\hline gggtggtttggttgg & 63 & $3.42 \times 10^{-8}$ & ggttggttttggttgg & 36 & $5.89 \times 10^{-7}$ \\
\hline \multicolumn{6}{|c|}{$\begin{array}{l}\mathrm{K}_{\mathrm{d}} \text { measurements of selected aptamers. Aptamer sequences are listed for } 15 \text { mer and } 16 \text { mer sequences tested for their interactions with } \\
\text { thrombin (see main text for details). } 15 \text { mer sequences have higher counts and lower dissociation constants than } 16 \text { mer sequences, though } \\
\text { within the } 15 \text { mers the dissociation constant does not correlate directly to the read count. Listed } \mathrm{K}_{\mathrm{d}} \text { 's are an average of three measurements } \\
\text { *Previously published by Bock, et al. (5). }\end{array}$} \\
\hline
\end{tabular}

not correlate with the measured $\mathrm{K}_{\mathrm{d}}$ values suggesting that some short sequences may only function in the context of a larger sequence that itself may not require significant conservation. In such a case, it is possible to test the affinity of aptamers both in the short form identified by the ASKAS method, as well as in the longer, native, form in which sequences were initially exposed to the target.

As aptamer sequence lengths increase, the frequency at which they occur in the library decreases, which may lead to difficulty in identifying longer aptamers using the ASKAS method. However, it is anticipated that any aptamers that bind to the target with a higher affinity than other aptamers in the pool will be outliers within that particular k-mer length. Therefore it is important to identify any outliers at each $\mathrm{k}$-mer length.

Prospects for single-round aptamer selection

Traditional SELEX methods have produced aptamers to hundreds of targets from proteins to small molecules. In some cases, modified nucleotides have been incorporated into aptamers to improve their binding or increase their resistance to nucleases $(13,14)$. The major downside to using modified nucleotides is that the SELEX process requires an amplification step so that any modified nucleotide must be able to be both read and written by a polymerase. This polymerase requirement greatly reduces the number of potential modifications that can be included in aptamers because polymerases often have to be mutated in order to adapt to the modification being used. In contrast, the single round of selection described here only requires that a polymerase can recognize a modified nucleotide and incorporate the appropriate complementary standard nucleotide in the copied strand. The ability to incorporate modified nucleotides without requiring a mutant polymerase will increase the availability of modifications available and therefore the potential diversity of aptamer libraries in the future.

\section{Acknowledgments}

This work was supported by the Science and Engineering Institutes of A*STAR (S.H., S.B., J.S.), The Scripps Research Institute (J.S.), and The Skaggs Institute for Chemical Biology (B.Z., K.D.J.).

\section{Competing interests}

The authors declare no competing interests.

\section{References}

1. Tuerk, C. and L. Gold. 1990. Systematic evolution of ligands by exponential enrichment: RNA ligands to bacteriophage T4 DNA polymerase. Science 249:505-510.

2. Ellington, A.D. and J.W. Szostak. 1990. In vitro selection of RNA molecules that bind specific ligands. Nature 346:818-822.

3. Huizenga, D.E. and J.W. Szostak. 1995. A DNA aptamer that binds adenosine and ATP. Biochemistry 34:656-665.

\section{Future of BIOTECH OPTICS.}

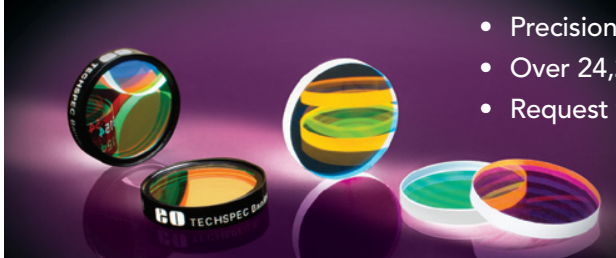

- Precision Filters, Aspheres, Objectives and more

- Over 24,200 Optics Available in Volume

Request your FREE catalog!

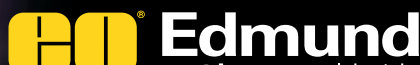

856.547.3488 | www.edmundoptics.com
4. Jhaveri, S. and A. Ellington. 2002. In vitro selection of RNA aptamers to a small molecule target. Curr Protoc Nucleic Acid Chem Chapter 9:Unit 95.

5. Bock, L.C., L.C. Griffin, J.A. Latham, E.H. Vermaas, and J.J. Toole. 1992. Selection of singlestranded DNA molecules that bind and inhibit human thrombin. Nature 355:564-566.

6. Lupold, S.E., B.J. Hicke, Y. Lin, and D.S. Coffey 2002. Identification and characterization of nuclease-stabilized RNA molecules that bind human prostate cancer cells via the prostate-specific membrane antigen. Cancer Res. 62:4029-4033.

7. Cho, M., Y. Xiao, J. Nie, and R. Stewart. a.T. Csordas, S.S. Oh, J.a. Thomson, and H.T. Soh. Quantitative selection of DNA aptamers through microfluidic selection and high-throughput sequencing. Proceedings of the National Academy of Sciences 107:15373-15378.

8. Kupakuwana, G.V., J.E. Crill, M.P. McPike, and P.N. Borer. 2011. Acyclic Identification of Aptamers for Human alpha-Thrombin Using Over-Represented Libraries and Deep Sequencing. PLoS One 6(5):e19395.

9. Tasset, D.M., M.F. Kubik, and W. Steiner. 1997. Oligonucleotide inhibitors of human thrombin that bind distinct epitopes. J. Mol. Biol. 272:688-698.

10. Kurtz, S., A. Narechania, J.C. Stein, and D. Ware. 2008. A new method to compute K-mer frequencies and its application to annotate large repetitive plant genomes. BMC Genomics 9:517.

11. Edgar, R.C. 2010. Search and clustering orders of magnitude faster than BLAST. Bioinformatics 26:2460-2461.

12. Berezovski, M.V., M.U.Musheev, A.P. Drabovich, J.V. Jitkova, and S.N. Krylov. 2006. Non-SELEX: selection of aptamers without intermediate amplification of candidate oligonucleotides. Nat. Protocols 1:1359-1369.

13. Latham, J.A., R. Johnson, and J.J. Toole. 1994. The application of a modified nucleotide in aptamer selection: novel thrombin aptamers containing 5-(1-pentynyl)-2'-deoxyuridine. Nucleic Acids Res. 22:2817-2822.

14. Gold, L., D. Ayers, J. Bertino, C. Bock, A. Bock E.N. Brody, J. Carter, A.B. Dalby, et al. 2010. Aptamer-based multiplexed proteomic technology for biomarker discovery. PLoS One 5:e15004.

Received 04 August 2011; accepted 03 November 2011

Address correspondence to Jonathan Scolnick, The Scripps Research Institute, La Jolla, CA, USA. email: jscolnick@gmail.com

To purchase reprints of this article, contact: biotech niques@fosterprinting.com 